\title{
Simultaneous Broadening Analysis of Multiple Bragg Edges Observed by Wavelength-resolved Neutron Transmission Imaging of Deformed Low-carbon Ferritic Steel
}

\author{
Hirotaka SATO, ${ }^{1) *}$ Kenji IWASE, ${ }^{2)}$ Takashi KAMIYAMA ${ }^{1)}$ and Yoshiaki KIYANAGI ${ }^{3)}$ \\ 1) Faculty of Engineering, Hokkaido University, Kita-13 Nishi-8, Kita-ku, Sapporo, Hokkaido, 060-8628 Japan. \\ 2) College of Engineering, Ibaraki University, 4-12-1 Nakanarusawa-cho, Hitachi, Ibaraki, $316-8511$ Japan. \\ 3) Graduate School of Engineering, Nagoya University, Furo-cho, Chikusa-ku, Nagoya, Aichi, 464-8603 Japan.
}

(Received on October 10, 2019; accepted on November 28, 2019; J-STAGE Advance published date: January 17, 2020)

\begin{abstract}
With the goal of real-space mapping of dislocation information using a wavelength-resolved (spectroscopic) neutron transmission imaging method, broadenings of multiple Bragg-edges in neutron transmission spectrum were investigated in detail for the first time. Data of time-of-flight (TOF) neutron transmission imaging and diffraction experiments on a polycrystalline low-carbon ferritic steel sample while undergoing tensile testing were analysed. The Bragg-edge neutron transmission spectroscopy was combined with the classical Williamson-Hall method corrected by the crystal elastic anisotropy using the ratio of diffraction Young's modulus, namely, the corrected classical Williamson-Hall (ccWH) method. As a result, the broadening values evaluated from the ccWH analysis of Bragg-edge data were consistent with results of both our TOF neutron diffraction experiments and previous reports. In addition, it was deduced that the line-broadenings appearing in the plastic deformation condition during tensile testing in our experiment were mainly caused by micro-strain (dislocation density) effect and not by crystallite size effect. Finally, a Bragg-edge broadening mapping method, using a simultaneous multiple Bragg-edges profile analysis based on the ccWH method, could identify plastically deformed zones in the sample more clearly than a traditional single Bragg-edge broadening analysis method.
\end{abstract}

KEY WORDS: pulsed neutron transmission imaging; multiple Bragg-edges broadening analysis; corrected classical Williamson-Hall method; micro-strain; dislocation density; crystallite size.

\section{Introduction}

Wavelength-resolved neutron transmission imaging using Bragg-edge (or Bragg-dip) profile analysis is a powerful tool used for crystallographic/metallurgical characterization because it can non-destructively and quantitatively visualize position-dependent crystalline microstructural information over a large area in a bulk material. Thus far, various Bragg-edge/dip profile analysis methods have been developed. For example, crystalline phase, ${ }^{1-3)}$ crystallographic texture, ${ }^{4-7}$ crystallite size from the primary extinction effect, ${ }^{6)}$ macro-strain ${ }^{8-10)}$ and crystalline grain orientation ${ }^{11-13)}$ have been extracted from wavelength-dependent neutron Bragg-edge/dip transmission data. Furthermore, the quantity of martensite phase in a quenched ferritic steel has been deduced from the broadening of a single Bragg-edge due to high micro-strain, high dislocation density and fine crystallite size. ${ }^{14)}$ In association with the single Bragg-edge broadening mapping, the plastically deformed zones in a ferritic steel plate undergoing tensile testing have been investigated,

\footnotetext{
* Corresponding author: E-mail: h.sato@eng.hokudai.ac.jp DOI: https://doi.org/10.2355/isijinternational.ISIJINT-2019-656
}

and the result has been compared with mapping results of the crystallographic texture evolution and the extinction effect-based crystallite size obtained from the Bragg-edge neutron transmission spectrum. ${ }^{15)}$ However, there were no studies which investigated about the broadening profile of multiple Bragg-edges systematically.

It is well known that line-broadening/profile of diffraction peaks are affected by crystal lattice defects. Thus far, micro-strain, dislocation density and crystallite size of a crystalline material have been quantitatively evaluated by diffraction peak profile analysis. For example, a single peak analysis method, ${ }^{16)}$ the classical Williamson-Hall (WH) method ${ }^{17,18)}$ and the classical Warren-Averbach (WA) method ${ }^{19)}$ have been proposed and applied in materials science. A multiple peaks analysis method such as the WH method and the WA method is useful for separately analysing micro-strain/dislocation density broadening and crystallite size broadening. Furthermore, the combination of the modified WH method and the modified WA method, the $\mathrm{mWH} / \mathrm{mWA}$ method, considering the dislocation contrast factor ${ }^{20)}$ has been proposed not only as a more correct analysis of dislocation density but also for the analysis of dislocation arrangements and dislocation edge/screw char- 
acter. $^{21)}$ The CMWP (convolutional multiple whole profile) fitting method which is based on the $\mathrm{mWH} / \mathrm{mWA}$ method has been also developed as one of the most reliable and useful data analysis methods. ${ }^{22)}$ On the other hand, Takaki et al. have reported that the corrected classical WH method, the ccWH method, in which the crystal elastic anisotropy is corrected by the ratio of diffraction Young's modulus, is usable for dislocation density evaluation of a low-carbon ferritic steel. $^{23-26)}$ We applied the ccWH method in our feasibility study because the sample condition was similar to that of Takaki's study, although the goal in the future is to apply the $\mathrm{mWH} / \mathrm{mWA}$ method. In any case, various evaluation methods for micro-strain, dislocation density/arrangement/ character and crystallite size, using the diffraction peak line-broadening/profile analysis, have been proposed and applied in materials science and engineering.

The combination of the analysis methods for diffractometry mentioned above with the Bragg-edge neutron transmission imaging method promises to be a powerful tool for investigating the information of dislocation characteristics. This is because it has several advantages for real-space analysis of a crystalline material, high spatial resolution, large area mapping and bulk analysis etc. Therefore, we have studied the broadenings of multiple Bragg-edges in detail for investigating the possibility of dislocation analysis, and newly developed and tested an effective Bragg-edge broadening analysis method as follows:

- Investigation of broadening, FWHM (full width at half maximum), of multiple Bragg-edges for diffraction indices $110,200,211,220,310$ and 321. Comparison of them with those of neutron diffraction peaks from the same crystalline grains (the same scattering vector) in the same polycrystalline sample. The goal was to check that the same broadenings were evaluated by both methods. We also aimed to confirming that the same data analysis method as used in diffractometry could be used in the Bragg-edge transmission method.

- Evaluation of the validity of the ccWH plot, its slope and intercept parameters, relating to micro-strain, dislocation density and crystallite size.

- Development of a new Bragg-edge profile fitting analysis method based on the ccWH model; the simultaneous broadening analysis method for multiple Bragg-edges.

- Demonstration of real-space mapping of parameters of the ccWH plot obtained from the simultaneous broadening analysis method of multiple Bragg-edges.

\section{Time-of-flight Neutron Transmission Imaging and Diffraction Experiments}

We conducted wavelength-resolved neutron experiments of both transmission imaging for observing multiple Bragg-edges and diffraction for observing multiple diffraction peaks. We used time-of-flight (TOF) spectroscopy at an accelerator-driven pulsed neutron source. We conducted in-situ neutron experiments during a tensile test of a low-carbon ferritic steel plate, in which broadenings of Bragg-edges and diffraction peaks were observed due to plastic deformation. In this section, the experimental conditions are summarized although our previous studies ${ }^{15,27)}$ reported about the experiment in detail.

\subsection{Sample}

Figure 1 shows a photograph of the tensile-tested sample with information on the dimensions. A $5 \mathrm{~mm}$ thick JIS-SS400 plate (ferrite $(\alpha$-Fe) with perlite; polycrystal; low-carbon steel; the yield point is larger than $245 \mathrm{~N} / \mathrm{mm}^{2}$; the crystalline grain size is several-ten micrometres ${ }^{6)}$ ) was used for tensile testing. The tensile loads were $0 \mathrm{kN}$ for determination of the initial Bragg-edge and diffraction peak profile, 10, 20, 25, $27.5,30,32.5,40,49 \mathrm{kN}$ and $20 \mathrm{~N}$ (load release condition). The estimated average stresses at the centre region of the sample were 66.6, 133.3, 166.6, 183.3, 200, 216.6, 266.6, 326.6 and $0.13 \mathrm{~N} / \mathrm{mm}^{2}$ where the cross-section area of the centre region was $30 \mathrm{~mm} \times 5 \mathrm{~mm}\left(150 \mathrm{~mm}^{2}\right)$. Hence, there was possibility that plastic deformation was caused at the 40 $\mathrm{kN}$ tensile or more because the estimated average stress might exceed the yield point. The tensile direction corresponded to the horizontal direction (see Fig. 1). The stress-strain curves at some positions of the sample were measured by strain gauge, and the results were described in Ref. 27.

The sample had two notches in the central region. In our previous studies, we observed that there were stress concentration zones near these notches. For example:

- The macro-strain (elastic-strain) increased near two notches up to the $32.5 \mathrm{kN}$ tensile, and then such high macrostrain regions expanded over the whole region after the 32.5 $\mathrm{kN}$ tensile. ${ }^{27)}$

- Crystallographic texture evolution, a cancellation of the primary extinction effect which reflects fine crystallites, and 110 Bragg-edge broadening were observed near two notches only at the $49 \mathrm{kN}$ tensile and the load release condition. $^{15)}$

Since it was thought that the plastic deformation was caused at the $49 \mathrm{kN}$ tensile, we analysed the broadenings of multiple Bragg-edges and diffraction peaks.

\subsection{Wavelength-resolved Neutron Transmission Imag- ing and Diffraction Measurements}

Figure 2 shows schematic layouts of wavelength-resolved (a) neutron transmission imaging and (b) neutron diffraction experiments performed at BL19 engineering materials diffractometer TAKUMI in Materials and Life Science Experimental Facility (MLF) in the Japan Proton Accelerator

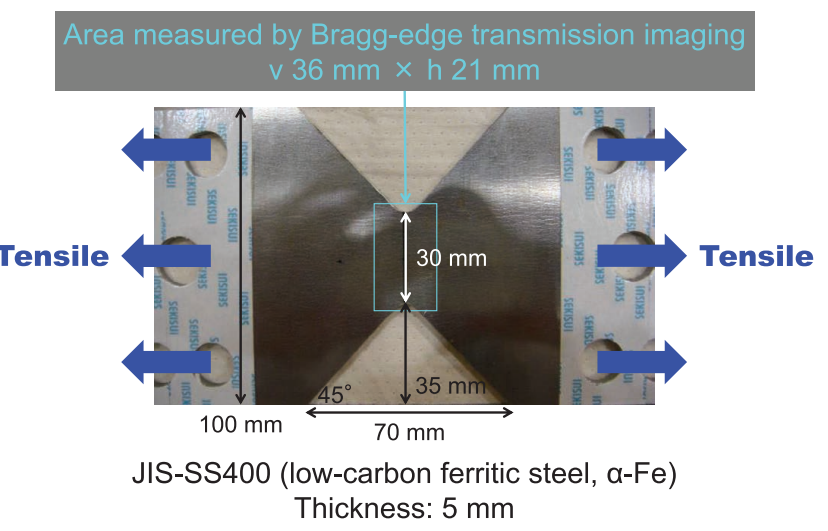

Fig. 1. Photograph of a measured ferritic steel sample and its dimensions. The region measured by the Bragg-edge neutron transmission imaging mode is shown. The sample had two notches where stress concentration zones existed. ${ }^{15,27)}$ (Online version in color.) 
Research Complex (J-PARC). ${ }^{28)}$ The TAKUMI instrument was connected to the poisoned-decoupled supercritical para$\mathrm{H}_{2}$ cold neutron moderator. Neutrons were transported to the instrument through the curved (upstream) and collecting (downstream) supermirror guide-tubes. The sample was set at the $40 \mathrm{~m}$ position away from the neutron source.

The sample was set for tensile testing, and compressive stress was induced along the normal direction of the sample plate (along the neutron transmission direction). The direction of the incident neutron beam was fixed. We set a transmission imaging detector behind the sample, and both were placed together on the tensile machine. On the other hand, since the diffraction detector bank of TAKUMI was fixed at the position of $2 \theta=90^{\circ}$ where $2 \theta$ is diffraction angle, the sample and also the transmission imaging detector need to be rotated by $45^{\circ}$ (see Fig. 2) in order to measure the same diffraction information from the same crystalline grains, namely the same scattering vector whose direction corresponds to the compression direction in the sample, in both the transmission imaging and diffraction modes.

The Bragg-edge neutron transmission imaging data were measured over a horizontal width of $20 \mathrm{~mm}$ which was set by the width of a $\mathrm{B}_{4} \mathrm{C}$ slit. The neutron TOF-imaging detector used was a $16 \times 16$ channels ${ }^{6} \mathrm{Li}$ glass scintillator pixel type detector. ${ }^{29)}$ The scintillator thickness was $1 \mathrm{~mm}$, and the detection efficiency for cold neutrons was about $95 \%$. The pixel size was $3 \mathrm{~mm}$, and the detectable area was $48 \mathrm{~mm} \times 48 \mathrm{~mm}$ which was larger than the beam size. The detector was placed at the $0.06 \mathrm{~m}$ position behind the sample. The transmission imaging data were measured in a range of 12 pixels $(36 \mathrm{~mm})$ in the vertical $\times 7$ pixels $(21$ $\mathrm{mm}$ ) in the horizontal directions as shown in Fig. 1.

The Bragg-edge neutron transmission data for comparison with the diffraction data were selected from the imaging data, and the used pixels were extracted from a central part of the sample, namely, 10 pixels $(30 \mathrm{~mm})$ in the vertical $\times$ 1 pixel $(3 \mathrm{~mm})$ in the horizontal directions, which corresponded to the neutron-irradiated height in the sample and was only $1 \mathrm{~mm}$ narrower in width than the slit width in the diffraction measurement.

The diffraction data were measured through a slit of 4 $\mathrm{mm}$ width and $40 \mathrm{~mm}$ height. The north detector bank was used without using the radial collimators of the TAKUMI diffractometer. The distance from the sample was $2.06 \mathrm{~m}$.

During the experiment, the $3 \mathrm{GeV}$ proton synchrotron was operated at $120 \mathrm{~kW}$. The disk chopper (frame overlap chopper) placed at a $7.7 \mathrm{~m}$ distance from the neutron source was set in $25 / 2 \mathrm{~Hz}$ (double frame) mode. As a result, neutrons up to $0.7 \mathrm{~nm}$ wavelength could be utilized. The TOF channel width was $5 \mu$ s for both the transmission imaging mode and the diffraction mode. The measurement time for the transmission imaging mode was 3 hours, and the measurement time for the diffraction mode was 5 minutes.

\subsection{Comparison of Neutron Spectra of Bragg-edge Transmission with Diffraction}

Here, we compared the Bragg-edge transmission spectra obtained over a $30 \mathrm{~mm} \times 3 \mathrm{~mm}$ area with the diffraction patterns obtained over a $30 \mathrm{~mm} \times 4 \mathrm{~mm}$ area as shown in Fig. 3. The relationship of the neutron wavelength $\lambda$, neutron flight time $t$ and crystal lattice plane spacing ( $d$-spacing) $d$ are described by the following equation:

$$
\lambda=\frac{h}{m\left(\frac{l}{t}\right)}=2 d \sin \theta .
$$

Here, $h$ is Planck's constant, $m$ is the neutron static mass, $l$ is the neutron flight path length from the neutron source to the detector (40.06 $\mathrm{m}$ for Bragg-edge transmission; 42.06 $\mathrm{m}$ for diffraction), and $\theta$ is the Bragg angle (one half of the scattering angle; $90^{\circ}$ for Bragg-edge transmission; $45^{\circ}$ for diffraction), respectively. As found by our previous work, ${ }^{15)}$ Fig. 3(a) clearly shows a change of transmission value over the whole wavelength region when measured at the $49 \mathrm{kN}$ tensile. This was caused by a change of texture and crystallite size (primary extinction effect) due to plastic deforma- (a)

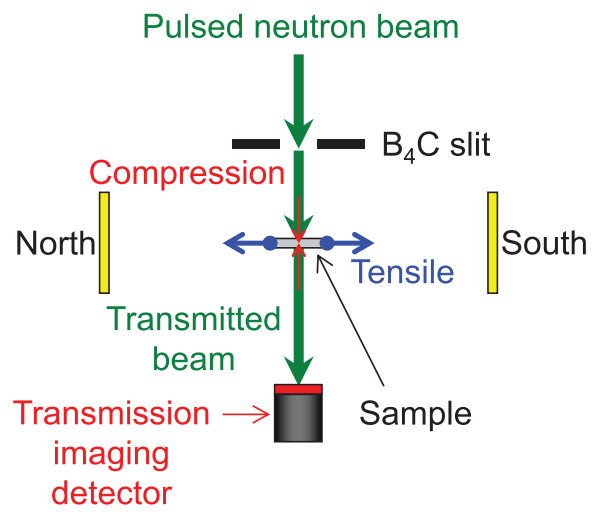

- Slit: 20 mm (width), 40 mm (height)

- Distance from sample to detector: $0.06 \mathrm{~m}$

- Measurement time: 3 hours (b)

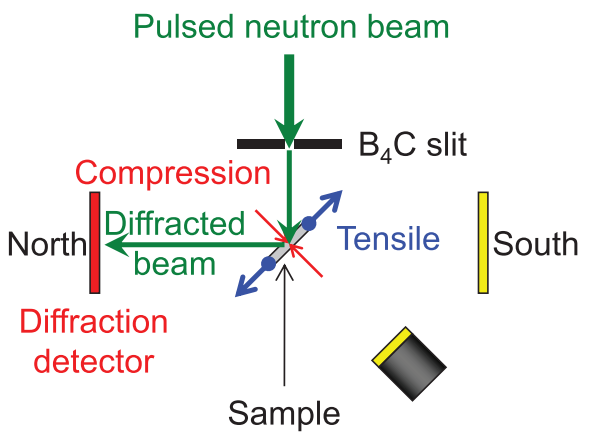

- Slit: $4 \mathrm{~mm}$ (width), $40 \mathrm{~mm}$ (height)

- Distance from sample to detector: $2.06 \mathrm{~m}$

- Measurement time: 5 minutes

Fig. 2. Schematic layouts of the pulsed neutron (a) transmission imaging and (b) diffraction experiments. (Online version in color.) 
(a)

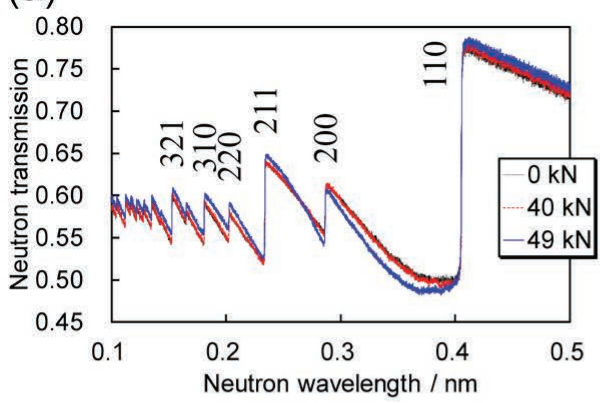

(c)

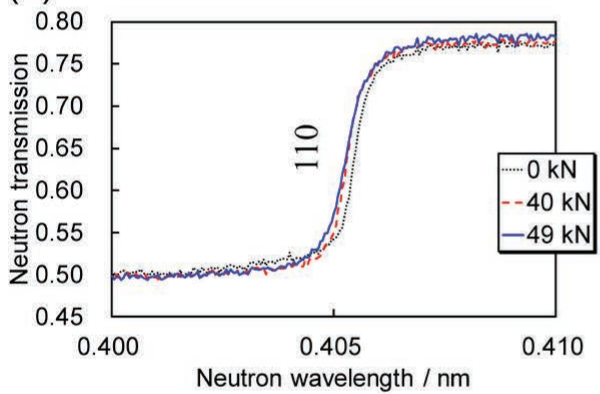

(e)

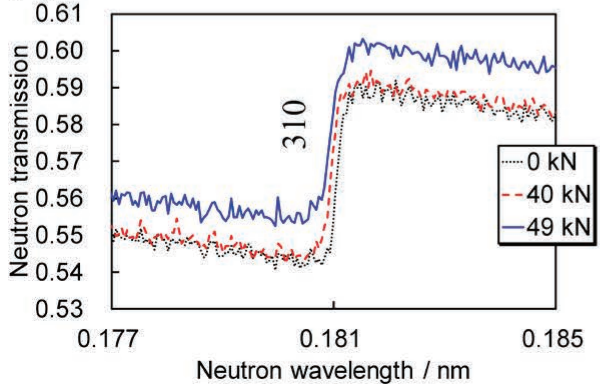

(b)

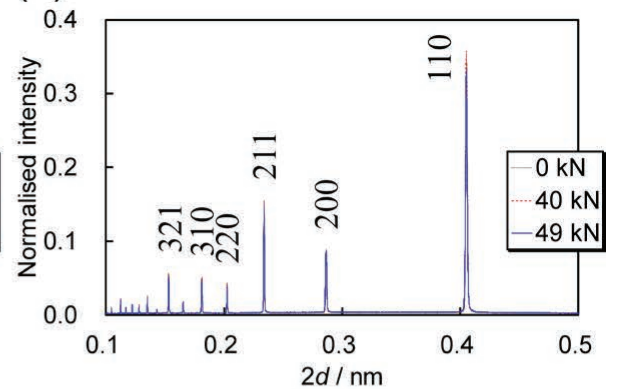

(d)

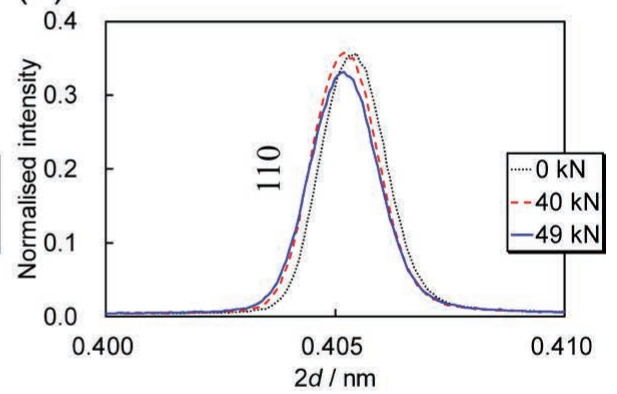

(f)

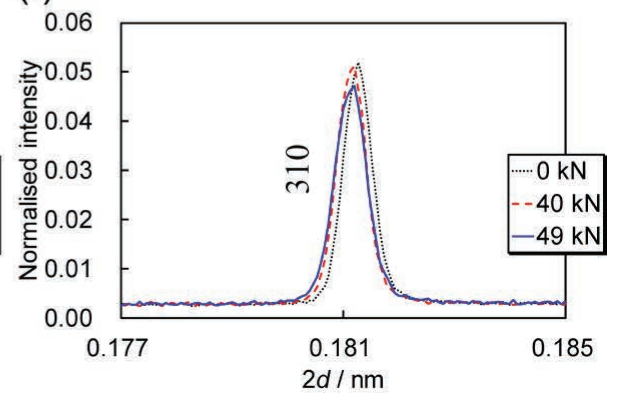

Fig. 3. Measured neutron spectra at the tensile load of $0 \mathrm{kN}$ (initial), $40 \mathrm{kN}$ (before plastic deformation) and $49 \mathrm{kN}$ (after plastic deformation). (a) Whole pattern of Bragg-edge transmission data obtained over $30 \mathrm{~mm} \times 3 \mathrm{~mm}$. (b) Whole pattern of diffraction data obtained over $30 \mathrm{~mm} \times 4 \mathrm{~mm}$. (c) 110 Bragg-edge profile. (d) 110 diffraction peak profile. (e) 310 Bragg-edge profile. (f) 310 diffraction peak profile. (Online version in color.)

tion. As found by our previous works, ${ }^{15,27)}$ Figs. 3(c)-3(f) shows that both Bragg-edge data and diffraction peak data simply shifts to shorter $d$-spacing without any profile change up to $40 \mathrm{kN}$. Then, broadenings of Bragg-edge and diffraction peak were observed due to the plastic deformation at the $49 \mathrm{kN}$ tensile. In our present study, we investigated such broadening changes of multiple diffraction indices in detail; not just a single diffraction index as in previous studies.

\section{Classical Williamson-Hall Plot with the Crystal Elastic Anisotropy Correction Using the Ratio of Diffraction Young's Modulus}

In this section, we show that the Bragg-edge broadenings of diffraction indices 110, 200, 211, 220, 310 and 321 are consistent with those of the corresponding diffraction peaks. Then, we also show that the corrected classical Williamson-Hall (ccWH) plots ${ }^{25}$ of the Bragg-edge data are proportional to the magnitude of the scattering vector corrected by the ratio of the diffraction Young's modulus. Finally, we discuss the validity of parameters obtained from the ccWH plot, by comparing with the results of previous diffraction studies.

\subsection{Comparison of FWHM between Bragg-edges and Diffraction Peaks Based on the Classical Williamson-Hall (cWH) Plot \\ Figure 4 shows examples of the profile fitting analysis} results of Bragg-edge data and diffraction peak data. We obtained the average value of $d$-spacing distribution, $d$, and FWHM (full width at half maximum) of the $d$-spacing distribution, $\Delta d$, by the profile fitting analysis. Figure 5 shows results of classical WH (cWH) plots of both Bragg-edge broadening and diffraction peak broadening at the $49 \mathrm{kN}$ tensile. Here, $k$ in the $\mathrm{cWH}$ plot means the magnitude of the scattering vector,

$$
k=\frac{2 \sin \theta}{\lambda}=\frac{1}{d}
$$

Then, $\Delta k$ in the cWH plot is the FWHM of the $d$-spacing distribution expressed in $k$ space, and can be calculated by the equation,

$$
\Delta k=\frac{\Delta d}{d^{2}-\frac{(\Delta d)^{2}}{4}} .
$$

This equation is derived from the relation between $d$ space 
and $k$ space as follows. $d_{-}$and $k_{-}$are assumed as a position of the left-hand side of half width at half maximum. $d_{+}$and $k_{+}$are assumed as a position of the right-hand side of half width at half maximum. Here, $\Delta k$ is equal to $k_{+}-k_{-} . k_{+}$is $1 / d_{-}$, and $k_{-}$is $1 / d_{+} \cdot d_{-}$is $d-\Delta d / 2$, and $d_{+}$is $d+\Delta d / 2$. Equation (3) can be derived by using these relationships.

FWHM of the $d$-spacing distribution, $\Delta d$, does not include the broadening component due to the instrumental resolution. For this reason, in our feasibility study, the broadening component due to the instrumental resolution was deduced from the experimental data of the $0 \mathrm{kN}$ tensile. Of course, in future actual use, a more suitable standard sample should be used for a more correct determination of the instrumental resolution function. The method of evaluating $d$ and $\Delta d$ from a Bragg-edge was proposed by our previous work. ${ }^{14)}$ The detailed equations and the procedure were described in a previous paper. ${ }^{30)}$ In the data analysis, the $d$-spacing distribution due to only the sample was assumed to be a Gaussian distribution $^{14,30)}$ although the instrumental resolution function was assumed to be of the Jorgensen-type asymmetric Bragg-edge profile function. ${ }^{1)}$ On the other hand, we extracted $d$ and $\Delta d$ from a diffraction peak through a simple profile fitting method using a Gaussian $d$-spacing distribution with a straight background baseline (see Fig. 4(d)). In the diffraction case, $\Delta d$ was obtained by the equation,

$$
\Delta d=\sqrt{\Delta d_{\text {measure }}^{2}-\Delta d_{0 \mathrm{kN}}^{2}}
$$

Here, $\Delta d_{\text {measure }}$ is the FWHM of a target diffraction peak data at a certain tensile load, and $\Delta d_{0 \mathrm{kN}}$ is FWHM of a diffraction peak at the $0 \mathrm{kN}$ tensile.

Firstly, we confirmed that the calculation procedures for both $k$ and $\Delta k$ were correct. This is because the $k$-dependence of $\Delta k$ at $0 \mathrm{kN}$ tensile $\left(\Delta d_{0 \mathrm{kN}}\right)$ in Fig. 5 corresponds to the reference instrumental resolution data shown by a previous work. $^{31)}$ Secondly, Fig. 5 shows that the $k$-dependence of the $\Delta k$ from the Bragg-edge is consistent with that from diffraction. The straight lines fitted to the cWH plots had the following characteristics:

$$
\begin{aligned}
& \begin{aligned}
\Delta k=0.0014 k+0.0006 \\
\text { for the cWH plot of Bragg - edge } \\
\text { transmission in Fig. } 5
\end{aligned} \\
& \Delta k=0.0013 k+0.0018 \\
& \text { for the cWH plot of diffraction in Fig. } 5
\end{aligned}
$$

Significant cWH plots could be observed only at the $49 \mathrm{kN}$ tensile and the load release conditions. Namely, significant line-broadenings did not appear up to the $40 \mathrm{kN}$ tensile as seen by our previous work $^{15)}$ which conducted single line-broadening analysis although we return to this point

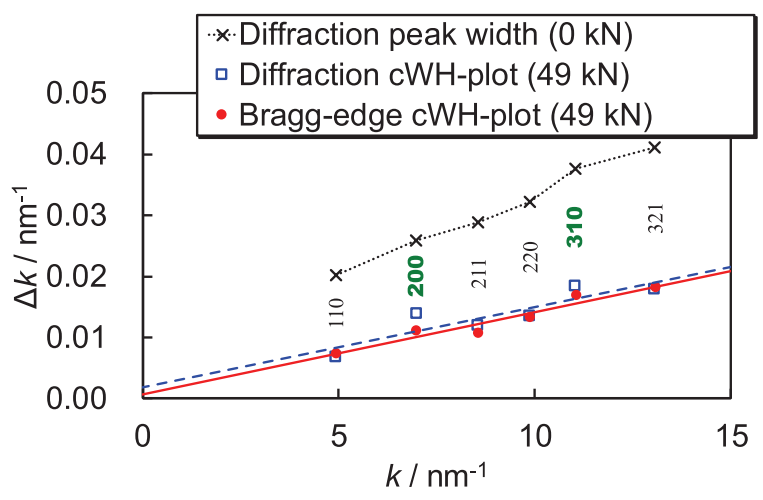

Fig. 5. cWH plots of neutron Bragg-edge transmission and diffraction data after the plastic deformation (tensile load of $49 \mathrm{kN}$ ). The solid and dashed lines are the fitted straight lines for each plot; solid line for Bragg-edge; dashed line for diffraction. (Online version in color.)
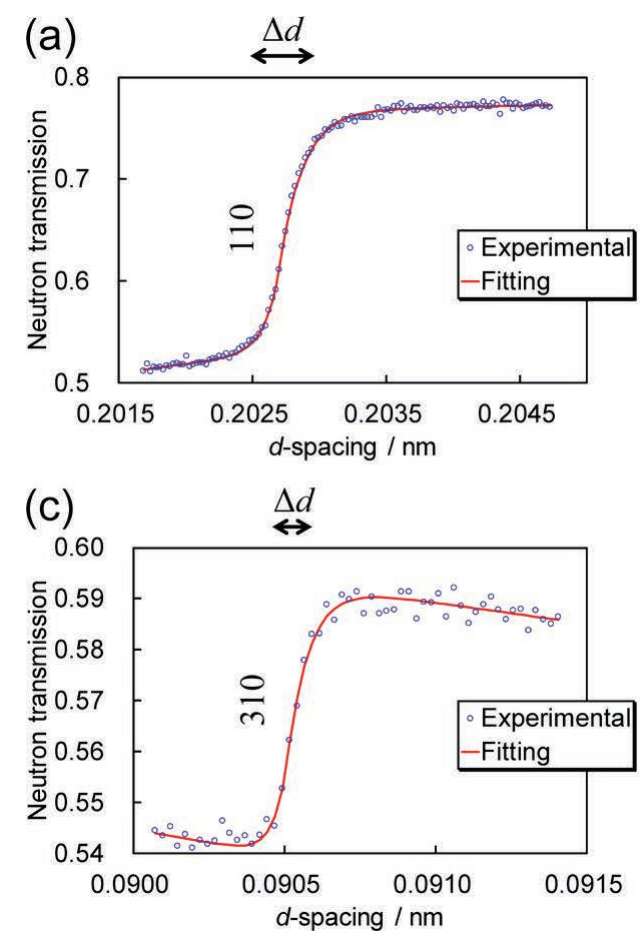

(b)

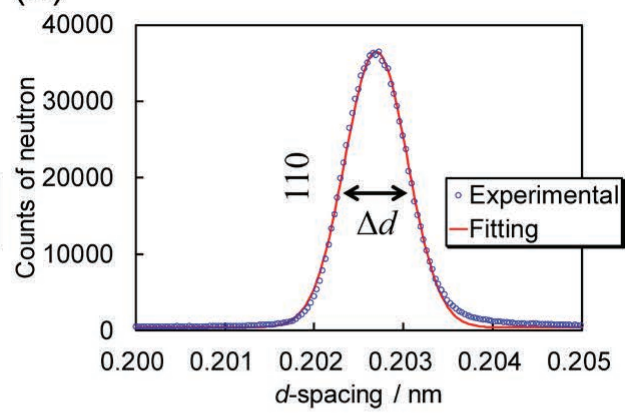

(d)

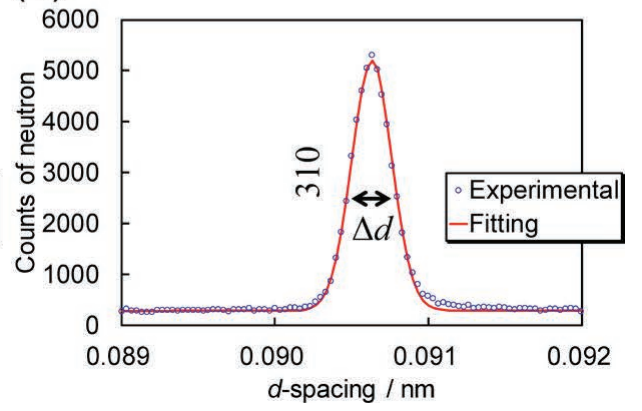

Fig. 4. Examples of the profile fitting analysis for single Bragg-edge of (a) 110 and (c) 310, and for single diffraction peak of (b) 110 and (d) 310, at the $0 \mathrm{kN}$ tensile. (Online version in color.) 
again in Sec. 4. In any case, in the broadening analysis using multiple diffraction indices, it was confirmed that the Bragg-edge transmission method could use the same principles of data analysis as used in the diffractometry.

\subsection{Corrected Classical Williamson-Hall (ccWH) Plots of Neutron Bragg-edge Transmission and Diffrac- tion Data}

The cWH plot can be ideally expressed by a straight line as a function of $k$,

$$
\Delta k=\varepsilon k+\alpha,
$$

where $\varepsilon$ (the slope of the cWH plot) is micro-strain, $\alpha$ (the intercept of the cWH plot) is $0.9 / D$ where $D$ is the size of spherical crystallite. ${ }^{17,18)}$ Thus, the micro-strain $\varepsilon$ relating to dislocation density, and crystallite size $D$ can be evaluated from the cWH plot. However, actual cWH plots are not generally a straight line due to the crystal elastic anisotropy depending on the diffraction lattice plane. Actually, due to the crystal elastic anisotropy, values of $\Delta k$ of 200 and 310 in the diffraction data clearly exist at the upper side of the straight dashed line (see Fig. 5). Thus, a mWH plot has been proposed as a correction using the dislocation contrast factor. ${ }^{21)}$

On the other hand, another approach, the corrected cWH (ccWH) plot, was proposed by Takaki et al. ${ }^{25)}$ They have suggested that this method was usable for dislocation density evaluation of a deformed low-carbon ferritic steel. ${ }^{23,24,26)}$ For a low-carbon ferritic steel with a grain size of severaltens of micrometres, the calculation procedure of dislocation density from the ccWH plot has been investigated by experimental studies using both TEM (transmission electron microscope) and X-ray diffraction. ${ }^{23,24,26)}$ These sample conditions are suitable for our experiment. For these reasons, we applied Takaki's methods to the Bragg-edge data for quantitative evaluation of broadening parameters.

The ccWH plot is expressed by

$$
\Delta k=\varepsilon\left(\frac{k}{\omega_{h k l}}\right)+\alpha
$$

Here, $\omega_{h k l}$ is the ratio of the diffraction Young's modulus. ${ }^{25)}$ Table 1 shows theoretical values of $\omega_{h k l}$ of each diffraction index. ${ }^{25}$ ) These were calculated by the Kröner model ${ }^{32)}$ and the elastic stiffness coefficients. ${ }^{33)}$ Takaki et al. tested the ccWH plots using Eq. (8), and they found that $\Delta k$ was clearly proportional to $k / \omega_{h k l}{ }^{25)}$ In other words, the crystal elastic anisotropy included in the cWH plot can be corrected by using $\omega_{h k l} . \omega_{h k l}$ is treated as the contrast factor as well as the dislocation contrast factor in the $\mathrm{mWH}$ plot.

Figure 6 shows the ccWH plot, converted from Fig. 5 and Table 1 . Thanks to the elastic anisotropy correction, the $\Delta k$ plots of 200 and 310 diffraction data are clearly closer to the fitted straight line than they were in the cWH plots. The fitted lines are expressed as follows:

$$
\begin{aligned}
\Delta k=0.0012\left(k / \omega_{h k l}\right)+0.0005 \\
\\
\text { for the ccWH plot of Bragg-edge } \\
\text { transmission in Fig. } 6
\end{aligned}
$$

$$
\Delta k=0.0013\left(k / \omega_{h k l}\right)+0.0003
$$

for the ccWH plot of diffraction in Fig. 6
Table 1. Theoretical values of the ratio of diffraction Young's modulus, $\omega_{h k l}$, for polycrystalline $\alpha$-Fe. ${ }^{25}$ )

\begin{tabular}{ccccccc}
\hline$h k l$ & 110 & 200 & 211 & 220 & 310 & 321 \\
\hline$\omega_{h k l}$ & 0.984 & 0.734 & 0.984 & 0.984 & 0.808 & 0.984 \\
\hline
\end{tabular}

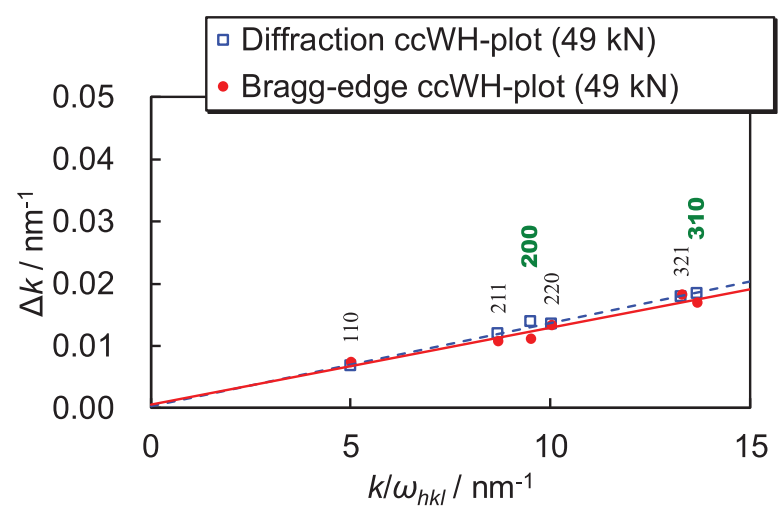

Fig. 6. ccWH plots of neutron Bragg-edge transmission and diffraction data after the plastic deformation (the tensile load of $49 \mathrm{kN}$ ). The solid and dashed lines are the fitted straight lines with each plot; solid line for Bragg-edge; dashed line for diffraction. (Online version in color.)

$\varepsilon$ (slope) and $\alpha$ (intercept) of the ccWH plots are slightly changed from ones of the cWH plots. In any case, the ccWH plots of both Bragg-edge transmission and diffraction coexist on almost the same straight line.

\subsection{Evaluation of Parameters Obtained from the ccWH Plots}

A conversion equation to calculate the dislocation density from the micro-strain $\varepsilon$ was proposed by based on TEM and $\mathrm{X}$-ray diffraction experimental studies for a low-carbon ferritic steel. ${ }^{23,24,26)}$ According to them, the dislocation density $\rho$ is calculated by the equation,

$$
\rho=9.3 \times\left(\frac{\varepsilon}{b}\right)^{2}=1.5 \times 10^{20} \varepsilon^{2}
$$

Here, $b$ is the magnitude of the Burgers vector of $\alpha$-Fe, $\sqrt{3} / 2 \times 0.28665 \mathrm{~nm}=0.24825 \mathrm{~nm}$. Here, $0.28665 \mathrm{~nm}$ is lattice constant of $\alpha$-Fe, and $\sqrt{ } 3$ is derived from $\sqrt{ }\left(h^{2}+k^{2}+l^{2}\right)$ where the vector direction $\langle h k l\rangle$ of the BCC crystal structure corresponds to $\langle 111\rangle$. The coefficient 9.3 was refined by Takaki's works ${ }^{23,24,26)}$ although so far other coefficients have been proposed, $16.1^{18)}$ and $6 \pi .^{34)}$ These were based on experiences that the dislocation density was proportional to the squared value of micro-strain for $\alpha$-Fe.

Hereafter, we evaluate parameters $\varepsilon, 1.5 \times 10^{20} \varepsilon^{2}, \alpha$ and $0.9 / \alpha$ obtained from the ccWH plot of Bragg-edges. At the $49 \mathrm{kN}$ tensile, we could draw a significant ccWH plot in Bragg-edge transmission. Here, the evaluated values are as follows; $\varepsilon=0.0012,1.5 \times 10^{20} \varepsilon^{2}=2.2 \times 10^{14} \mathrm{~m}^{-2}, \alpha=$ $0.0005 \mathrm{~nm}^{-1}$ and $0.9 / \alpha=1800 \mathrm{~nm}$. These parameters are compared with results of previous diffraction studies below.

Takaki et al. reported the slope $\varepsilon$ of the ccWH plot of a cold-rolled low-carbon $(0.0056 \% \mathrm{C})$ ferritic steel with a 50 $\mu \mathrm{m}$ grain size. ${ }^{25)}$ The condition of the sample is close to that of our present study, and the results are comparable. Takaki et al. reported that $\varepsilon$ in their experiments was distributed 
from 0.0005 to $0.0020 .{ }^{25)}$ Our results $(\varepsilon=0.0012)$ exist within the range of these results. The validity of the $1.5 \times$ $10^{20} \varepsilon^{2}$ value is discussed in Sec. 4.2.

Takaki et al. reported that the intercept $\alpha$ of the ccWH plot in their experiments was distributed from $0.0006 \mathrm{~nm}^{-1}$ to $0.0030 \mathrm{~nm}^{-1} .^{25)}$ Our results $\left(\alpha \sim 0.0005 \mathrm{~nm}^{-1}\right)$ are close to this range. However, the $\alpha$ value in our present study is very small, and hence $0.9 / \alpha$ in our present study, 1800 $\mathrm{nm}$, is very large as a crystallite size evaluated by linebroadening, compared with previous reports; for example, the largest crystallite size case, $600 \mathrm{~nm}$, was reported by Kato et $a l .{ }^{35)}$ This is because the JIS-SS400 sample used has large grains of several-tens of micrometres in size, ${ }^{6)}$ and it is likely that the broadening effect due to crystallite size $(\alpha)$ was very small. Another possibility is that use of the $\Delta d_{0 \mathrm{kN}}$ data as approximation of instrumental resolution is not sufficiently reasonable for line-broadening analysis relating to crystallite size effect. For these reasons, we supposed that the crystallite size obtained from the line-broadening analysis was not significant in our present study. In other words, it was deduced that the Bragg-edge broadening at the $49 \mathrm{kN}$ tensile was caused by micro-strain/dislocation effect and not by crystallite fining effect. This is further discussed in Sec. 4.2.

It is worth nothing that the large crystallite size of JIS-SS400 could probably be accurately evaluated by the primary extinction effect. $\left.{ }^{6}\right)$ This means that the crystallite size evaluation using the Bragg-edge broadening analysis is best applied to relatively fine crystallites, while primary extinction analysis is better suited to relatively coarse crystallites.

\section{Analysis of ccWH Plot's Parameters Using Simulta- neous Broadening Analysis of Multiple Bragg-edges}

When attempting a profile analysis of Bragg-edge data with low statistics as in a pixel-by-pixel analysis, the evaluated each $\Delta k$ may have a large statistical error. In such a case, high accuracy ccWH plots cannot be obtained, and then analysis of parameters of the ccWH plot (the slope $\varepsilon$ and the intercept $\alpha$ ) is difficult. In addition, such an individual Bragg-edge profile analysis is time-consuming. To avoid this problem, we have developed a simultaneous profile fitting analysis method for multiple Bragg-edges, based on the ccWH model, using the same Bragg-edge transmission data used in the previous section.

\subsection{Simultaneous Broadening Analysis of Multiple Bragg-edges Using the ccWH Model-based Profile Fitting Method}

Although Steuwer et al. developed a simultaneous multiple Bragg-edges fitting analysis (the Pawley-type analysis) method for the purposes of lattice parameter refinement, ${ }^{36)}$ the aim of the present analysis is to use the Bragg-edge broadening analysis to refine the parameters of the ccWH model. With this approach, a higher statistical quality of analysis is achieved than that possible by the single Bragg-edge analysis; further discussion is described in Sec. 5.2.

Figure 7 shows an example of the results of the simultaneous profile fitting analysis applied to multiple Bragg-edges (110, 200, 211, 220, 310 and 321) obtained by the Bragg-edge neutron transmission measurement at the 49 $\mathrm{kN}$ tensile. The details of the procedure in the developed data analysis program are as follows:

- All TOF-dependent neutron transmission data are converted to data as a function of $k$.

- Before the simultaneous multiple Bragg-edges profile fitting analysis, instrumental resolution functions of 110 , 200, 211, 220, 310 and 321, which are described by the Jorgensen-type edge-profile function, are determined by individual single Bragg-edge profile fitting analysis of the data measured at the $0 \mathrm{kN}$ tensile.

- The target data, such as in Fig. 7, is analysed by simultaneous multiple Bragg-edges profile fitting. Then, the parameters of the ccWH model (the slope $\varepsilon$ and the intercept $\alpha$ ), which give the $\Delta k$ of each Bragg-edge by Eq. (8), are refined. Note that only the data around the Bragg-edges is used for fitting like the Pawley-type analysis. ${ }^{36)}$

Figure 7 shows that a reasonable profile fit was achieved for each Bragg-edge. Table 2 shows a comparison of the parameters of the ccWH plots from the individual analysis method (Fig. 6 and Eq. (9)) and the simultaneous analysis method (Fig. 7). The difference between them is at the same level as that between diffraction and Bragg-edge transmission (Fig. 6 and Eqs. (9) and (10)). The reason why the slope of the simultaneous method is smaller than that of the individual method and the intercept of the simultaneous method is larger than that of the individual method is explained as follows. In the simultaneous method, the fitting weights mainly exist in large Bragg-edges such as 110, 211 and 200. In Fig. 6, the 110, 211 and 200 datapoints in the ccWH plot of Bragg-edge transmission would produce a lower slope and larger intercept on their own than the total datapoints do in the entire ccWH plot. This causes the small differences shown in Table 2.

\subsection{Evaluation of Refinement Parameters of the ccWH Model Obtained by Simultaneous Multiple Bragg-edges Profile Analysis Method}

Figure 8 shows the tensile-load dependence of $\varepsilon, 1.5 \times$ $10^{20} \varepsilon^{2}, \alpha$ and $0.9 / \alpha$, obtained from the simultaneous multiple Bragg-edges broadening analysis. The refinement parameters were obtained under the restriction condition of "within positive value". $\varepsilon$ and $1.5 \times 10^{20} \varepsilon^{2}$ significantly increase from the $40 \mathrm{kN}$ tensile to the $49 \mathrm{kN}$ tensile, and the detectable $1.5 \times 10^{20} \varepsilon^{2}$ value is estimated as being on the order of $10^{14} \mathrm{~m}^{-2}$. This value is not so unusual since it was reported in a study evaluating a ferritic steel that the minimum dislocation density was around $10^{14} \mathrm{~m}^{-2}$. $^{34}$ )

On the other hand, $\alpha$ and $0.9 / \alpha$ did not indicate a significant dependence on the tensile load. As discussed in Sec. 3.3, since the measured ferritic steel sample had large crystalline grains, the intercept of the ccWH plot is too small (the crystallite size is too large) for the line-broadening analysis. For this reason, with respect to the ferritic steel sample used in the present study, it is difficult to discuss the tensile-load dependence of the crystallite size effect in line-broadening although the obtained $\alpha$ values are consistent with a similar previous study. ${ }^{25)}$ In other words, it is probable that the Bragg-edge broadening at the $49 \mathrm{kN}$ tensile was caused by micro-strain/dislocation effect but not by crystallite fining effect. Incidentally, as a point of interest, 

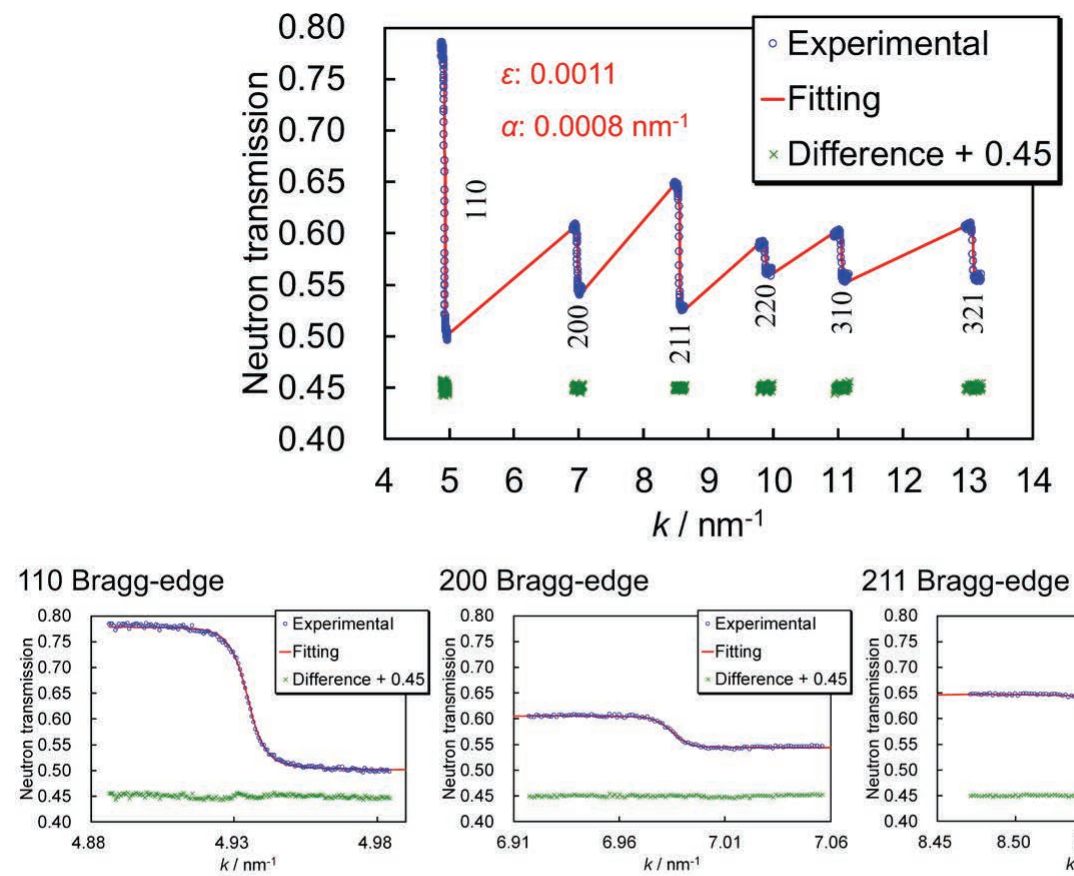

200 Bragg-edge

211 Bragg-edge
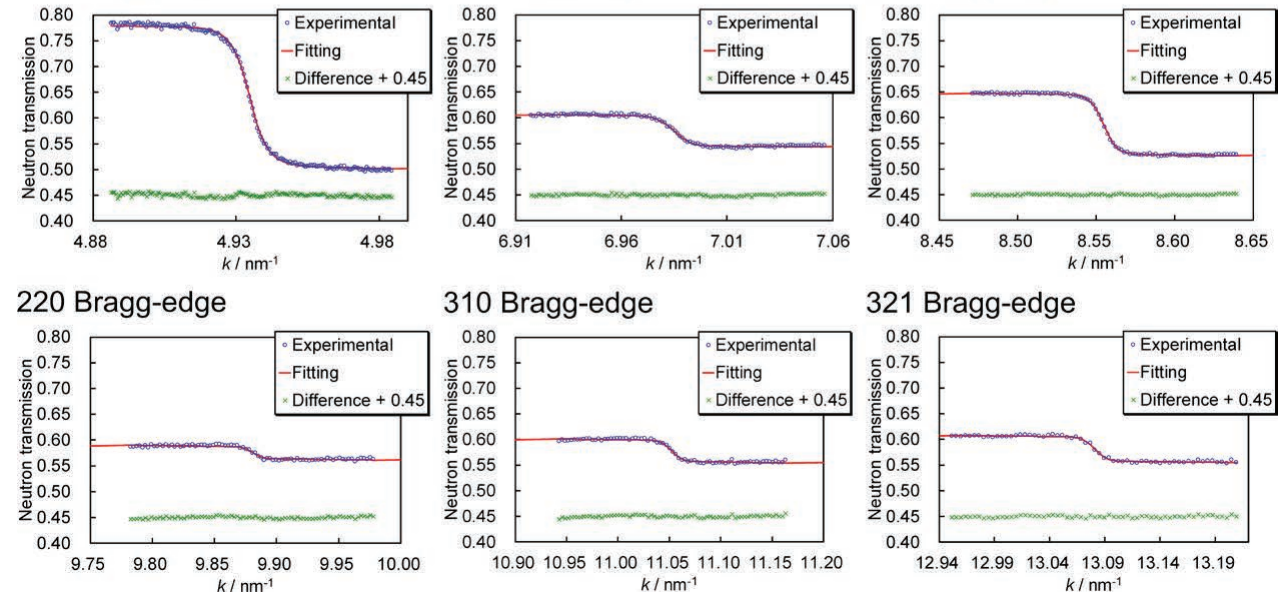

310 Bragg-edge

321 Bragg-edge
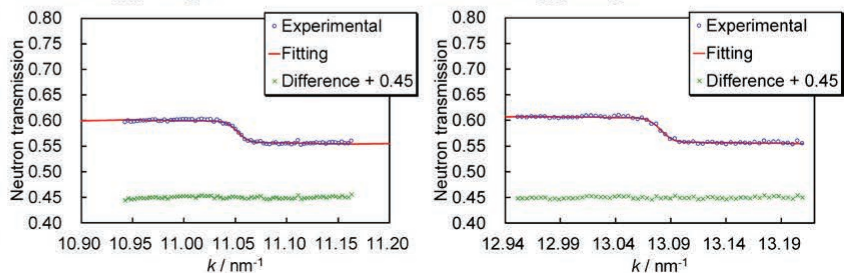

Fig. 7. A result of the simultaneous multiple Bragg-edges profile fitting based on the ccWH model. (Online version in color.)

Table 2. Comparison of refined parameters of the ccWH plot of the individual analysis method (Fig. 6) and the simultaneous analysis method (Fig. 7).

\begin{tabular}{ccccc}
\hline & Slope $\varepsilon$ & $1.5 \times 10^{20} \varepsilon^{2} / \mathrm{m}^{-2}$ & Intercept $\alpha / \mathrm{nm}^{-1}$ & $0.9 / \alpha / \mathrm{nm}$ \\
\hline $\begin{array}{c}\text { Individual } \\
\text { analysis }\end{array}$ & 0.0012 & $2.2 \times 10^{14}$ & 0.0005 & 1800 \\
$\begin{array}{c}\text { Simultaneous } \\
\text { analysis }\end{array}$ & 0.0011 & $1.9 \times 10^{14}$ & 0.0008 & 1200 \\
\hline
\end{tabular}

the crystallite size of this sample became significantly small at the $49 \mathrm{kN}$ tensile as determined by a primary extinction analysis. ${ }^{15)}$ Hence, we restricted attempts to map only the information relating to micro-strain and dislocation, as described in the next section.

\section{Mapping of Slope Parameter of the ccWH Plot}

\subsection{Real-space Distribution of the ccWH Plot's Slope Parameter Before and After the Plastic Deformation}

Figure 9 shows mapping results of the $1.5 \times 10^{20} \varepsilon^{2}$ value at the tensile loads $0 \mathrm{kN}, 40 \mathrm{kN}, 49 \mathrm{kN}$ and $20 \mathrm{~N}$ (load release). These images were obtained by pixel-by-pixel analyses over the whole area observed as shown in Fig. 1. The Bragg-edge neutron transmission spectrum analysis method used was the simultaneous multiple Bragg-edges broadening analysis method based on the ccWH model.

As seen in Fig. $8\left(\right.$ b), the $1.5 \times 10^{20} \varepsilon^{2}$ value suddenly increases from $40 \mathrm{kN}$ to $49 \mathrm{kN}$. After the load release, the $1.5 \times 10^{20} \varepsilon^{2}$ value slightly decreases. Two regions of high $1.5 \times 10^{20} \varepsilon^{2}$ value (about $3 \times 10^{14} \mathrm{~m}^{-2}$ ) exist around two notches at the $49 \mathrm{kN}$ tensile. This trend is close to the findings of the previous study ${ }^{15}$ showing that the crystallographic texture evolution and fine crystallites are suddenly caused by the plastic deformation at two notches at the 49 $\mathrm{kN}$ tensile. In addition, after the observation of high $1.5 \times$ $10^{20} \varepsilon^{2}$ value's regions at the $49 \mathrm{kN}$ tensile, we discovered its emergent regions where relatively high $1.5 \times 10^{20} \varepsilon^{2}$ values $\left(2 \sim 7 \times 10^{13} \mathrm{~m}^{-2}\right)$ appear; see white arrows in Fig. 9(b). Such emergent phenomenon at the $40 \mathrm{kN}$ tensile is also observed in the $\varepsilon$ data shown in Fig. 8(a); see the black solid arrow in Fig. 8(a).

\subsection{Effectiveness of Simultaneous Multiple Bragg-edges Broadening Analysis}

Figures 9(c) and 9(d) show clearly plastic deformation zones near the two notches. However, these zones are less clear in the mapping results of the FWHM of the 110 $d$-spacing distribution at the $49 \mathrm{kN}$ tensile and the load release, which were obtained by the single Bragg-edge broadening analysis, as shown by Fig. 4 in Ref. 15. In addition, we could not observe two plastic-deformation zones by using the single Bragg-edge broadening analysis for 200 , 211, 220, 310 and 321 Bragg-edges. For these reasons, we can confirm that we can observe plastic deformation zones with low statistical error when using the simultaneous multiple Bragg-edges broadening analysis method. 
(a)

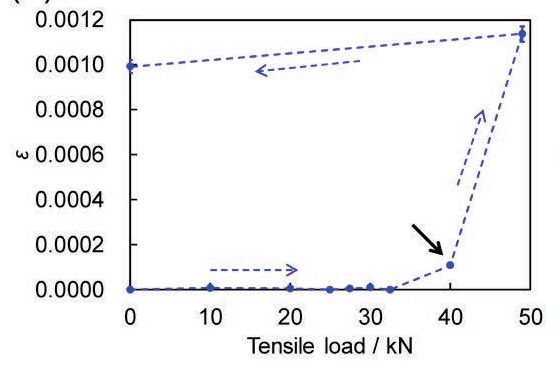

(c)

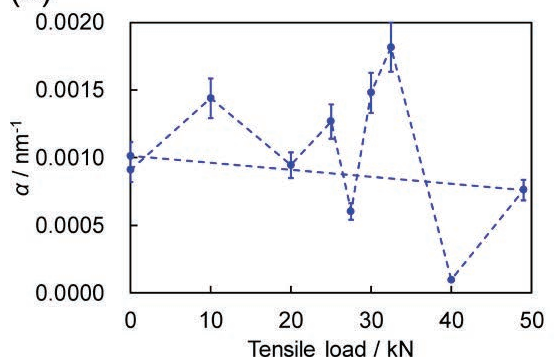

(b)

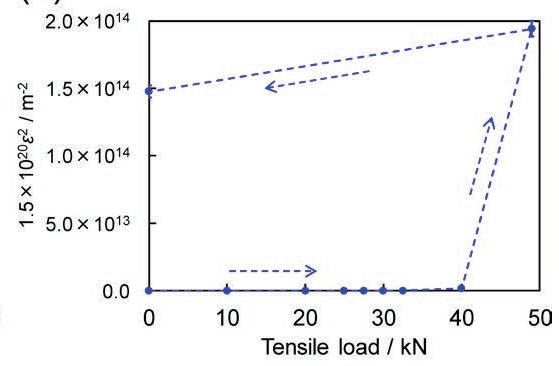

(d)

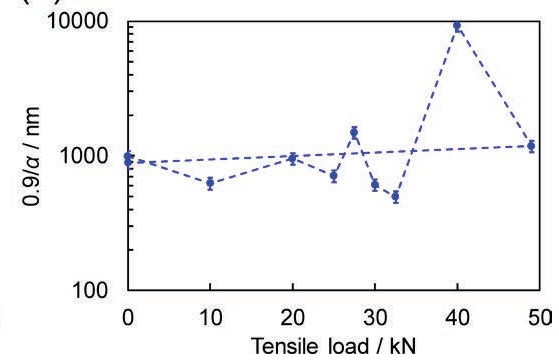

Fig. 8. Tensile-load dependence of (a) slope of the ccWH plot $\varepsilon$, (b) $1.5 \times 10^{20} \varepsilon^{2}$, (c) intercept of the ccWH plot $\alpha$ and (d) $0.9 / \alpha$, obtained by the simultaneous multiple Bragg-edges broadening analysis method. (Online version in color.)

(a) $0 \mathrm{kN}$

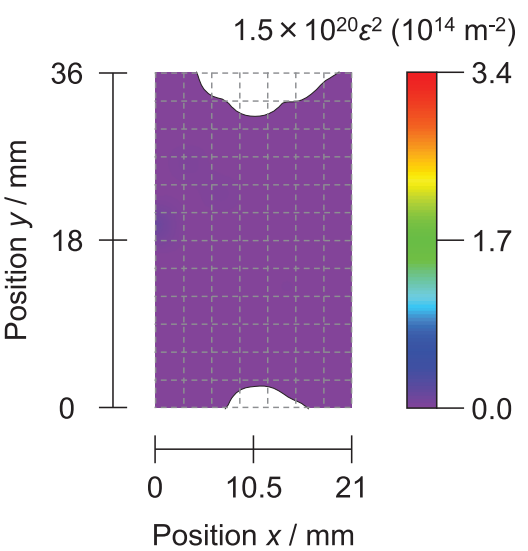

(c) $49 \mathrm{kN}$

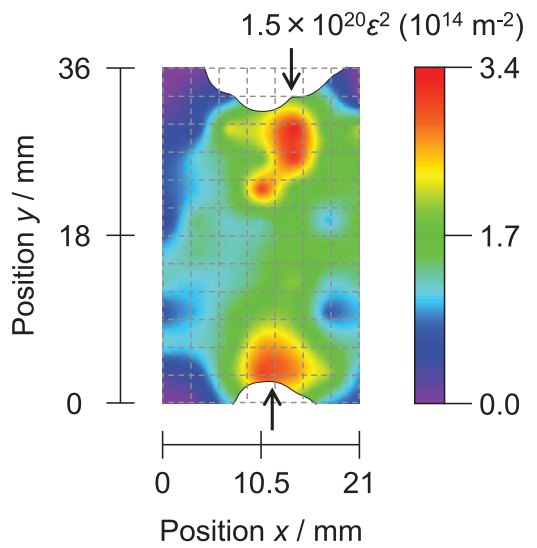

(b) $40 \mathrm{kN}$

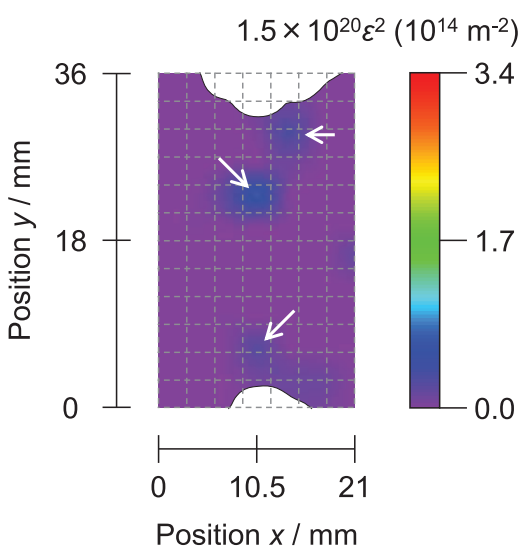

(d) Load release

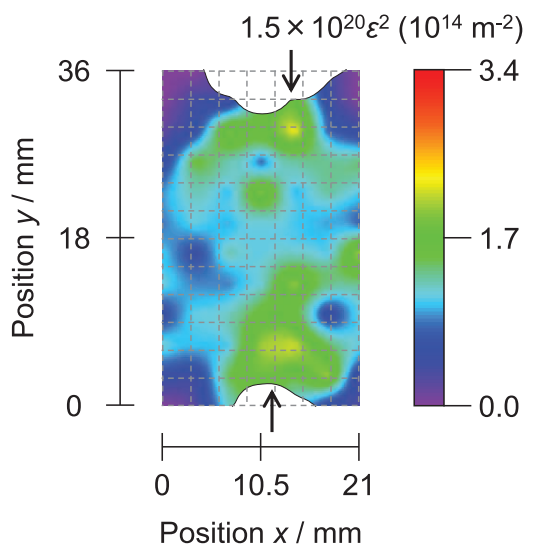

Fig. 9. Mapping results of the $1.5 \times 10^{20} \varepsilon^{2}$ value at the tensile load of (a) $0 \mathrm{kN}$, (b) $40 \mathrm{kN}$, (c) $49 \mathrm{kN}$ and (d) release, obtained by the pulsed neutron Bragg-edge transmission imaging method and its simultaneous multiple Braggedges broadening analysis based on the ccWH model. (Online version in color.)

\section{Conclusions}

We carried out the first feasibility study of a dislocation information mapping method using wavelength-resolved
Bragg-edge neutron transmission imaging. It was first confirmed that the same line-broadening analysis methodology used in diffractometry could be applied to the Bragg-edge transmission imaging method as the Bragg-edge broaden- 
ings were consistent with diffraction peak broadenings. Subsequently, we applied the ccWH method, the WH method including a crystal elastic anisotropy correction using the ratio of diffraction Young's modulus, to our data. The parameters of the ccWH plots, the slope relating to the dislocation density and the intercept relating to the crystallite size, were consistent with results of previous similar diffraction studies.

However, since the ccWH plots were not robust for low statistical neutron data such as the pixel-by-pixel data analysed in the Bragg-edge transmission imaging method, a reliable evaluation of parameters of the ccWH plot was difficult. Therefore, we developed a simultaneous multiple Bragg-edges profile fitting analysis method based on the ccWH model. As a result, we found a possibility that a dislocation density about $10^{14} \mathrm{~m}^{-2}$ was detected with some confidence. On the other hand, it was difficult to significantly evaluate the size effect of the large crystallites in the ferritic steel sample used in the present study. In other words, we concluded that the Bragg-edge broadening observed in this study was caused by micro-strain/dislocation effect and not by crystallite fining effect. Finally, we were able to perform mapping of slope parameter of the ccWH plot. As a result, plastic deformation regions (stress concentration regions) in the sample were visualized more clearly than when using the single Bragg-edge broadening analysis method.

In this study, we applied the ccWH model with the simultaneous multiple Bragg-edges profile fitting analysis method to a low-carbon ferritic steel sample. However, we expect that this methodology can be extended to a versatile and reliable dislocation analysis method like the $\mathrm{mWH} / \mathrm{mWA}$ model. $^{21)}$ It is similar to the CMWP method used in diffractometry. ${ }^{22)}$ Such an extension will provide information on dislocation density, dislocation arrangement and dislocation edge/screw character in the future. The findings of our present study indicate the feasibility of such future developments for a wavelength-resolved neutron Bragg-edge transmission imaging method.

\section{Acknowledgements}

The neutron experiments at J-PARC MLF BL19 "TAKUMI" were performed under a user program (Proposal No. 2009A0026). The authors thank Dr S. Harjo (JAEA J-PARC), Dr S. Takata (JAEA J-PARC), Dr T. Ito (CROSS) and Dr K. Aizawa (JAEA J-PARC) for experimental assistances.

\section{REFERENCES}

1) S. Vogel: Ph.D. thesis, Christian Albrechts Universität, (2000), https:// nbn-resolving.org/urn:nbn:de:gbv:8-diss-3306, (accessed 2008-5-23).

2) A. Steuwer, P. J. Withers, J. R. Santisteban and L. Edwards: J. Appl. Phys., 97 (2005), 074903. https://doi.org/10.1063/1.1861144

3) R. Woracek, D. Penumadu, N. Kardjilov, A. Hilger, M. Boin, J. Banhart and I. Manke: Adv. Mater., 26 (2014), 4069. https://doi. org/10.1002/adma.201400192

4) J. R. Santisteban, L. Edwards and V. Stelmukh: Phys. B, 385-386 (2006), 636. https://doi.org/10.1016/j.physb.2006.06.090

5) W. Kockelmann, G. Frei, E. H. Lehmann, P. Vontobel and J. R. Santisteban: Nucl. Instrum. Methods Phys. Res. Sect. A, 578 (2007),
421. https://doi.org/10.1016/j.nima.2007.05.207

6) H. Sato, T. Kamiyama and Y. Kiyanagi: Mater. Trans., 52 (2011), 1294. http://doi.org/10.2320/matertrans.M2010328

7) J. R. Santisteban, M. A. Vicente-Alvarez, P. Vizcaino, A. D. Banchik, S. C. Vogel, A. S. Tremsin, J. V. Vallerga, J. B. McPhate, E. Lehmann and W. Kockelmann: J. Nucl. Mater., 425 (2012), 218. https://doi.org/10.1016/j.jnucmat.2011.06.043

8) J. R. Santisteban, L. Edwards, M. E. Fitzpatrick, A. Steuwer, P. J. Withers, M. R. Daymond, M. W. Johnson, N. Rhodes and E. M. Schooneveld: Nucl. Instrum. Methods Phys. Res. Sect. A, 481 (2002), 765. https://doi.org/10.1016/S0168-9002(01)01256-6

9) A. S. Tremsin, J. B. McPhate, A. Steuwer, W. Kockelmann, A. M. Paradowska, J. F. Kelleher, J. V. Vallerga, O. H. W. Siegmund and W. B. Feller: Strain, 48 (2012), 296. https://doi.org/10.1111/ j.1475-1305.2011.00823.x

10) C. M. Wensrich, J. N. Hendriks and M. H. Meylan: Strain, 52 (2016), 80. https://doi.org/10.1111/str.12171

11) J. R. Santisteban: J. Appl. Crystallogr., 38 (2005), 934. https://doi. org/10.1107/S0021889805028190

12) F. Malamud and J. R. Santisteban: J. Appl. Crystallogr., 49 (2016), 348. https://doi.org/10.1107/S1600576716000443

13) H. Sato, Y. Shiota, S. Morooka, Y. Todaka, N. Adachi, S. Sadamatsu, K. Oikawa, M. Harada, S. Y. Zhang, Y. H. Su, T. Kamiyama, M. Ohnuma, M. Furusaka, T. Shinohara and Y. Kiyanagi: J. Appl. Crystallogr., 50 (2017), 1601. https://doi.org/10.1107/ S1600576717012900

14) H. Sato, T. Sato, Y. Shiota, T. Kamiyama, A. S. Tremsin, M. Ohnuma and Y. Kiyanagi: Mater. Trans., 56 (2015), 1147. https:// doi.org/10.2320/matertrans.M2015049

15) T. Kamiyama, K. Iwase, H. Sato, S. Harjo, T. Ito, S. Takata, K. Aizawa and Y. Kiyanagi: Phys. Procedia, 88 (2017), 50. https://doi. org/10.1016/j.phpro.2017.06.006

16) Th. H. de Keijser, J. I. Langford, E. J. Mittemeijer and A. B. P. Vogels: J. Appl. Crystallogr., 15 (1982), 308. https://doi.org/10.1107/ S0021889882012035

17) G. K. Williamson and W. H. Hall: Acta Metall., 1 (1953), 22. https:// doi.org/10.1016/0001-6160(53)90006-6

18) G. K. Williamson and R. E. Smallman: Philos. Mag., 1 (1956), 34. https://doi.org/10.1080/14786435608238074

19) B. E. Warren and B. L. Averbach: J. Appl. Phys., 21 (1950), 595. https://doi.org/10.1063/1.1699713

20) T. Ungár, I. Dragomir, Á. Révész and A. Borbély: J. Appl. Crystal$\operatorname{logr}$, 32 (1999), 992. https://doi.org/10.1107/S0021889899009334

21) T. Ungár and A. Borbély: Appl. Phys. Lett., 69 (1996), 3173. https:// doi.org/10.1063/1.117951

22) G. Ribárik, J. Gubicza and T. Ungár: Mater. Sci. Eng. A, 387-389 (2004), 343. https://doi.org/10.1016/j.msea.2004.01.089

23 ) D. Akama: Ph.D. thesis, Kyushu University, (2017), http://hdl.handle. net/2324/1807148, (accessed 2018-4-1).

$24)$ D. Akama, T. Tsuchiyama and S. Takaki: J. Soc. Mater. Sci. Jpn., 66 (2017), 522 (in Japanese). https://doi.org/10.2472/jsms.66.522

25) S. Takaki, F. Jiang, T. Masumura and T. Tsuchiyama: ISIJ Int., 58 (2018), 769. https://doi.org/10.2355/isijinternational.ISIJINT-2017-642

26) S. Takaki, T. Masumura, F. Jiang and T. Tsuchiyama: ISIJ Int., 58 (2018), 1181. https://doi.org/10.2355/isijinternational.ISIJINT-2018-021

27) K. Iwase, H. Sato, S. Harjo, T. Kamiyama, T. Ito, S. Takata, K. Aizawa and Y. Kiyanagi: J. Appl. Crystallogr., 45 (2012), 113. https://doi.org/10.1107/S0021889812000076

28) S. Harjo, T. Ito, K. Aizawa, H. Arima, J. Abe, A. Moriai, T. Iwahashi and T. Kamiyama: Mater. Sci. Forum, 681 (2011), 443. https://doi. org/10.4028/www.scientific.net/MSF.681.443

29) H. Sato, O. Takada, S. Satoh, T. Kamiyama and Y. Kiyanagi: Nucl. Instrum. Methods Phys. Res. Sect. A, 623 (2010), 597. https://doi. org/10.1016/j.nima.2010.03.082

30) H. Sato: J. Imaging, 4 (2018), 7. https://doi.org/10.3390/jimaging4010007

31) Y. Tomota, S. Sato and S. Harjo: Tetsu-to-Hagané, 103 (2017), 73 (in Japanese). https://doi.org/10.2355/tetsutohagane.TETSU-2016-085

32) H. Behnken and V. Hauk: Z. Metallkd., 77 (1986), 620.

33) A. E. Lord and D. N. Beshers: J. Appl. Phys., 36 (1965), 1620. https:// doi.org/10.1063/1.1703098

34) Y. Tomota, P. Lukas, S. Harjo, J.-H. Park, N. Tsuchida and D. Neov: Acta Mater., 51 (2003), 819. https://doi.org/10.1016/ S1359-6454(02)00473-1

35) T. Kato, S. Sato, Y. Saito, H. Todoroki and S. Suzuki: Adv. X-Ray Chem. Anal. Jpn., 47 (2016), 167 (in Japanese).

36) A. Steuwer, P. J. Withers, J. R. Santisteban, L. Edwards, G. Bruno, M. E. Fitzpatrick, M. R. Daymond, M. W. Johnson and D. Wang: Phys. Status Solidi A, 185 (2001), 221. https://doi.org/10.1002/1521-396X (200106)185:2<221::AID-PSSA221 > 3.0.CO;2-C 